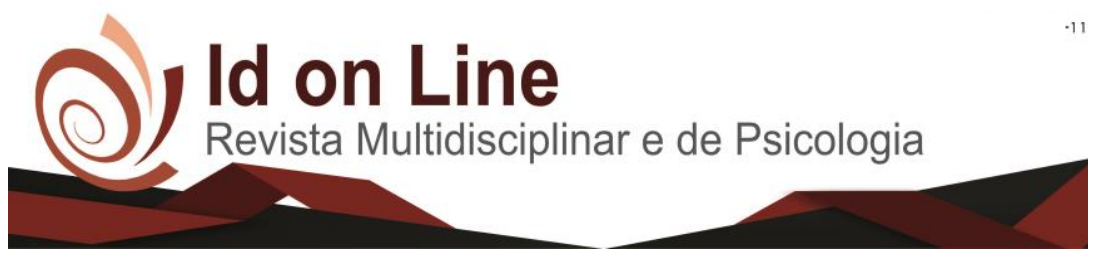

DOI: 10.14295/idonline.v13i46.1989

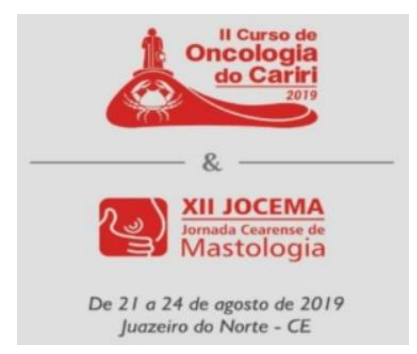

Resumo

\title{
SCREENING DE CÂNCER COLORRETAL NA POPULAÇÃO COM IDADE SUPERIOR A 75 ANOS
}

\author{
MENDES, Ana Carla da Silva ${ }^{1}$; MENDES, Isabelle Lima²; SOARES, Laryza Souza ${ }^{3}$; SILVA, Antônio Guilherme \\ Túlio $^{4}$; LIBÓRIO, Jaiany Rodrigues ${ }^{5}$; RODRIGUES, Hianca de Souza ${ }^{6}$.
}

Introdução: Câncer colorretal (CCR) é a $3^{\circ}$ neoplasia mais comum do mundo e $90 \%$ dos casos são em pessoas com mais de 50 anos. No Brasil, estimou-se 36360 novos casos em 2018. CCR apresenta curso lento, logo, o diagnóstico precoce aumenta a chance de cura. O rastreio ocorre através de exame de sangue oculto nas fezes (SOF) anual, retossigmoidoscopia a cada 5 anos e SOF a cada 3 e colonoscopia a cada 10. Há consenso sobre o início aos 50 anos, bem como, na presença de sinais e sintomas e iniciar antes para pessoas de alto risco, mas há divergência quanto terminar aos 75 anos. Objetivo: Observar se a triagem de CCR nos idosos, com mais de 75 anos, os benefícios superam aos riscos. Metodologia: Fez-se uma revisão sistemática baseada no Protocolo Prisma. A pergunta central foi elaborada a partir da determinação do PICO, que definiu população, intervenção, conflito e desfecho, indagando sobre a continuação do rastreamento de CCR em pessoas com idade maior que 75 anos possibilitando a melhoria da qualidade de vida. Usaram-se os descritores (Desc/BVS): "Colorectal Neoplasms", "Mass Screening" e "Aged" no PubMed(141), COCHRANE(79) e Goolge Scholar(1), com os filtros: ensaio-clínico, estudo observacional e últimos 5 anos, dos 221 trabalhos, incluiu-se 6 em inglês e português, datando de 2016 a 2018. Os critérios de inclusão foram: estudos concluídos, retratar todos os descritores e responder a pergunta-guia. Resultados: O rastreio não é recomendando para pacientes com mais de 75 anos ou com menos de 10 anos de expectativa de vida caso a colonoscopia anterior for negativa, mas fazer colonoscopia e SOF até 85 anos para quem não passou por triagem prévia. Um estudo com rastreio por SOF, CCR foi diagnosticado em $62 \%$ dos pacientes com 51-60 anos, em 60\% entre 61-70 anos e em 57\% 71-80 anos. Uma população de 1.355.692, entre 70 e 79 anos, na grupo de 75-79 anos, naqueles que realizaram a colonoscopia, efeitos adversos ocorreram em 10,3 a cada 1000 e uma redução de risco de CCR 3\% a 2,8\% de risco de CCR quando comparadas aos que não realizavam. Um estudo observacional comparou 3 grupos: 65-69 anos, 76-84 anos e 85-89 anos, notou que o rastreamento e o tratamento de CCR diminuía ao aumentar a idade e as comorbidades. Conclusão: O rastreio de CCR, em idosos maiores de 75 anos, deve ser uma decisão compartilhada entre médico e paciente, observando idade, estado de saúde e capacidade de tolerar os testes de triagem e intervenções, para que os benefícios superem os riscos.

Palavras-chave: Neoplasias Colorretais; Idoso; Programas de Rastreamento

\footnotetext{
${ }^{1}$ Autora; Acadêmica do curso de medicina na Faculdade de Medicina Estácio de Juazeiro do Norte.

e-mail: anademariaedejesus@gmail.com

${ }^{2}$ Orientadora; Docente na Faculdade de Medicina Estácio de Juazeiro do Norte; e-mail: isabellelimamendes@hotmail.com;

${ }^{3}$ Coautora; Acadêmica do curso de medicina na Faculdade de Medicina Estácio de Juazeiro do Norte.

e-mail: laryzasouza@gmail.com;

${ }^{4}$ Coautor; Acadêmico do curso de medicina na Faculdade de Medicina Estácio de Juazeiro do Norte.

e-mail: gtulio19@gmail.com;

${ }^{5}$ Coautora; Acadêmica do curso de medicina na Faculdade de Medicina Estácio de Juazeiro do Norte.

e-mail: hiancasouzarodrigues@gmail.com

${ }^{6}$ Coautora; Acadêmica do curso de medicina na Faculdade de Medicina Estácio de Juazeiro do Norte; e-mail: rodriguesjaiany@ gmail.com
} 


\section{Referências:}

BATISTA, T.F. et al.Achados Colonoscópicos em Idosos Extremos. 19a Semana de Pesquisa da Universidade Tiradentes. 2017. ISSN: 1807-2518

GARCÍA-ALBÉNIZ,X. et al. Effectiveness of Screening Colonoscopy to Prevent Colorectal Cancer Among Medicare Beneficiaries Aged 70 to 79 Years: A Prospective Observational Study. Ann Intern Med. 2017 Jan 3;166(1):18-26. DOI: 10.7326/M16-0758. Epub 2016 Sep 27.

KLABUNDE, C. et al. Influence of Age and Comorbidity on Colorectal Cancer Screening in the Elderly. American Journal of Preventive Medicin. 2016.2 51(3),e67e75. DOI:10.1016/j.amepre.2016.04.018

KWNAG-SIG, L. et al.Cost Effectiveness of Colorectal Cancer Screening Interventions with Their Effects on Health Disparity Being Considered. Cancer Res Treat; 48(3): 1010-9, 2016 Jul.

REX, D. K. et al. Colorectal Cancer Screening: Recommendations for Physicians and Patients from the U.S. Multi-Society Task Force on Colorectal Cancer. The American Journal of Gastroenterology. 2017. 112(7), 1016-1030. DOI:10.1038/ajg.2017.174.

TH, L. et al. Recommendations on prevention and screening for colorectal cancer in Hong Kong. Hong Kong Med J. 2018. Oct. 24(5):521-526. DOI: 10.12809/hkmj177095. 